\title{
Cryo-Electron Tomography Provides Insight into the Native Architecture of the Measles Virus Assembly Site
}

\author{
Joshua D. Strauss ${ }^{1}$, Zunlong, $\mathrm{Ke}^{2}$, Richard K. Plemper ${ }^{3}$, and Elizabeth R. Wright ${ }^{1}$ \\ ${ }^{1 .}$ Department of Pediatrics, Emory University School of Medicine, and Children's Healthcare of \\ Atlanta, Atlanta, Georgia, USA. \\ 2. School of Biology, Georgia Institute of Technology, Atlanta, Georgia, USA. \\ ${ }^{3 .}$ Institute for Biomedical Sciences, Georgia State University, Atlanta, Georgia, USA.
}

Measles virus $(\mathrm{MeV})$ is a pleomorphic, enveloped, single-stranded RNA virus, which assembles at the cell plasma membrane (PM). Details regarding the native arrangement of the $\mathrm{MeV}$ macromolecular complexes at the assembly site are lacking at the molecular level. Ultrastructural analysis of $\mathrm{MeV}$ by cryo-electron tomography (cryo-ET) has been limited to examinations of the purified virus [1, 2]. To gain insight into the process of virus assembly we utilized whole cellular cryo-ET to directly visualize $\mathrm{MeV}$ assembly sites in infected human-derived cells.

Subconfluent cultures of HeLa and MRC-5 cells (ATCC) were grown directly on gold Quantfoil TEM grids (Quantifoil Micro Tools GmbH, Jena, Germany). Cells were infected with MeV strains, including wild type and recombinant strains that were GFP labeled (recMeV-GFP MeV) [1] or contained an extension to the stalk region of the hemagglutinin $(\mathrm{H})$ protein (recMeV-H- $(181 \nabla 41 \times)[3]$ ) with a multiplicity of infection of 1,6 or 10 . The TEM grids were examined by phase-contrast light microscopy at 24-48 hours post infection to determine the optimal time for cryo-immoblization based on evidence of cytopathic effects. TEM grids were plunged into liquid ethane using a Cryoplunge 3 system (Gatan, Pleasanton, CA) as previously described [4]. Cryo-grids were imaged with a JEOL JEM-2200FS TEM (JEOL, Ltd., Japan) equipped with an in-column Omega energy filter, operated with a slit with of $20 \mathrm{eV}$. Bidirectional tilt-series, acquired at $2^{\circ}$ increments over an angular range of $-62^{\circ}$ to $+62^{\circ}$, were collected using SerialEM [5]. Images were recorded at a magnification of 20,000 $\times$, which corresponded to a pixel size of $2.94 \AA$ using a DE-20 direct electron detector camera (Direct Electron, LP, San Diego, CA) at 12 frames per second. Data was processed as previously described [3] and tomograms were reconstructed using the IMOD software package [6]. Tomographic reconstructions were binned by 4 and low pass filtered to enhance contrast.

Thin areas along the periphery of the infected cells were imaged by cryo-ET and revealed the organization of viral complexes, as illustrated in Fig 1. At assembly sites, the PM was thickened and appeared to be double-layered, thus, indicating the presence of the matrix $(\mathrm{M})$ protein. Regions of wellordered fusion $(\mathrm{F})$ and hemagglutinin $(\mathrm{H})$ glycoproteins were detected on the PM and were only present in areas that contained the underlying $M$ protein lattice. Of particular interest, we observed $M$ protein 2D paracrystalline arrays where spacing between the subunits was $7-8 \mathrm{~nm}$. We are investigating the hypothesis that the variation in $\mathrm{M}$ subunit spacing arises from its conformational flexibility, which enables the $M$ protein array to adapt to variations in PM curvature at sites of assembly. Direct visualization of $\mathrm{MeV}$-infected cells by cryo-ET has proved valuable for determining the native organization of the M protein, glycoproteins, and ribonucleoprotein (RNP) at the MeV assembly site [7]. 


\section{References:}

[1] MA Brindley, et al. Journal of Virology 87(21) (2014), p. 11693.

[2] L Liljeroos, et al. Proceedings of the National Academy of Sciences of the United States of America 108(44) (2011) p. 18085.

[3] T Paal, et al. Journal of Virology 83(20) (2009), p. 10480.

[4] JD Strauss, et al. Journal of Virology 90(3) (2015) p. 1507.

[5] DN Mastronarde. Journal of Structural Biology 152(1) (2005) p. 36.

[6] JR Kremer, DN Mastronarde, and JR McIntosh, Journal of Structural Biology 116(1)(1996), p. 71.

[7] This research was supported by funds from Emory University, Children's Healthcare of Atlanta, the Emory Center for AIDS Research, the Georgia Research Alliance, the James B. Pendleton Foundation, National Institutes of Health (5R21AI101775, 1R01GM114561, 3R01GM104540-03S1) to E.R.W, and (5R01A1083402) to R.K.P and (5F32GM112517) to J.D.S, and the National Science Foundation (0923395) to E.R.W, and (S10 RR025679) to P.W.S. All EM data was collected at the Emory University Robert P. Apkarian Integrated Electron Microscopy Core.

Figure 1. Cryo-ET of the native $\mathrm{MeV}$ assembly site in a MeV-infected MRC-5 cell. (A-F) Tomographic slices $(5.88 \mathrm{~nm})$ through the 3D volume. (A) The head domains of the $\mathrm{H}$ glycoprotein are visible and below them are (B) the F glycoprotein trimers. (C) The M protein forms an ordered 2D array, as seen in the enlarged inset of an unfiltered tomographic slice and corresponding Fourier transform. (D) The RNP located below the M layer (black triangles). M protein is not evenly distributed over the PM but forms patches as indicated in the enlarged inset. (E) The black triangles pointing to PM decorated with M protein and white triangles point to the PM. Scale bars 100 and $20 \mathrm{~nm}$.
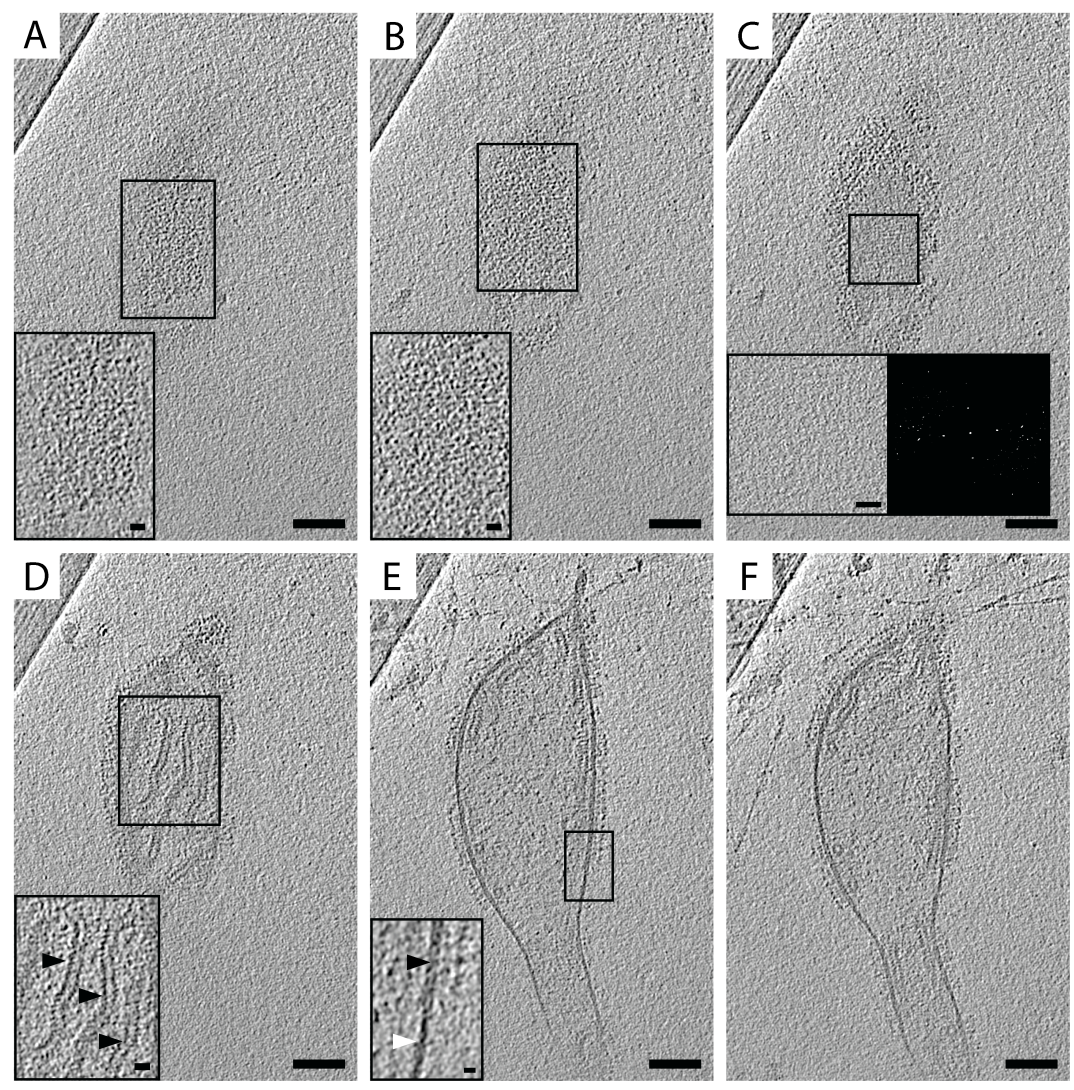